\title{
NATURAL CONVECTION AND RADIATION IN A RADIAL POROUS FIN WITH VARIABLE THERMAL CONDUCTIVITY
}

\author{
M.T. DARVISHI and F. KANI \\ Department of Mathematics, Razi University \\ Kermanshah 67149, IRAN \\ R.S.R. GORLA* \\ Department of Mechanical Engineering \\ Cleveland State University \\ Cleveland, Ohio, USA \\ E-mail: rama.gorla@yahoo.com
}

\begin{abstract}
In this study, the effects of radiation and convection heat transfer in a radial porous fin are considered. The geometry considered is that of a rectangular profile fin. The porous fin allows the flow to infiltrate through it and solid-fluid interaction takes place. This study is performed using Darcy's model to formulate the heat transfer equation. The thermal conductivity is assumed to be a function of temperature. The effects of the natural convection parameter $N c$, radiation parameter $N r$ and thermal conductivity parameter $m$ on the dimensionless temperature distribution and heat transfer rate are discussed. The results suggest that the radiation transfers more heat than a similar model without radiation.
\end{abstract}

Key words: porous radial fin, natural convection, radiation, thermal performance, spectral collocation method.

\section{Introduction}

Finned surfaces are used to improve the performance of heat exchangers. Optimization of the size and cost of fins is an important target of fin designer. Some engineering applications require lighter fins with a higher rate of heat transfer. In such applications, high thermal conductivity metals are used. However, the cost of high thermal conductivity metals is also high. Thus, the enhancement of heat transfer can be achieved by increasing the heat transfer rate and decreasing the size and cost of fin. The major heat transfer from the surface to the surrounding fluid takes place by convection process. Increasing the heat transfer mainly depends on the heat transfer coefficient, surface area available and the temperature difference between the surface and surrounding fluid. Recently, porous fins have been used for increasing heat transfer. The basic philosophy behind using porous fins is to increase the effective area through which heat is transported to the ambient fluid. Extensive research has been done in this area and many references are available especially for heat transfer in porous fins.

Kiwan (2006) conducted a thermal analysis of natural convection porous fins. Gorla and Bakier (2011) investigated natural convection and radiation in porous fins. Abu-Hijleh (2003) studied the effect of using porous fins on the forced convection heat transfer from a horizontal cylinder. Yu and Chen (1999) performed a study on optimization of a circular fin with variable thermal parameters. Nguyen and Aziz (1992) compared the heat transfer rates from convecting-radiating fins for different profile shapes. Kundu and Bhanja (2007) determined analytically the performance and optimum dimensions of T-shape fins with

\footnotetext{
* To whom correspondence should be addressed
} 
variable thermal conductivity and convective heat transfer. Kundu and Bhanja (2010) presented an optimum design analysis of porous fins. Mobedi and Sunden (2006) studied the natural convection heat transfer due to a thermal heat source located in a vertical plate fin.

The studies listed above clearly show the work on porous fins has been confined to straight fins of rectangular profile and pin fins. No attempt has been made to study porous radial fins although radial fins are used in numerous applications where porous fins can provide a lighter, cheaper and superior alternative to solid metallic fins. The purpose of this paper is to analyze the thermal performance of a radial porous fin of uniform thickness. The energy equation appears in the form of an ordinary differential equation containing two nonlinear terms. The first nonlinear term is associated with the buoyancy effects in the fluid and the permeability of the porous medium. The second nonlinearity is a consequence of radiative cooling at the surface of the fin. We assume the base of the fin at a constant temperature and the tip of the fin to be adiabatic. The effective thermal conductivity is assumed to be a function of temperature. The present formulation characterizes the effect of ambient temperature differently from Gorla and Bakier (2011) and provides a better picture of the effect of the ambient temperature on the thermal performance of the fin.

\section{Mathematical analysis}

Consider a radial (annular or circular) fin of base radius $r_{b}$, tip radius $r_{t}$ and thickness $t$ as illustrated in Fig.1. The base of the fin is maintained at a constant temperature $T_{b}$. The tip of the fin is assumed to be adiabatic. The fin is made of a porous material of effective thermal conductivity $k_{\text {eff }}$ and permeability $K$. The fin is in contact with an ambient fluid which infiltrates through the fin following Darcy's law. The fluid has a specific heat $c_{p, f}$, density $\rho_{f}$, kinematic viscosity $v_{f}$, thermal conductivity $k_{f}$ and coefficient of volumetric expansion $\beta_{f}$. The exposed surfaces (top and bottom) are assumed to be a gray medium with a constant emissivity $\varepsilon$ and emit radiation to the ambient fluid (temperature $T_{b}$ ), which also serves as the radiation heat sink.

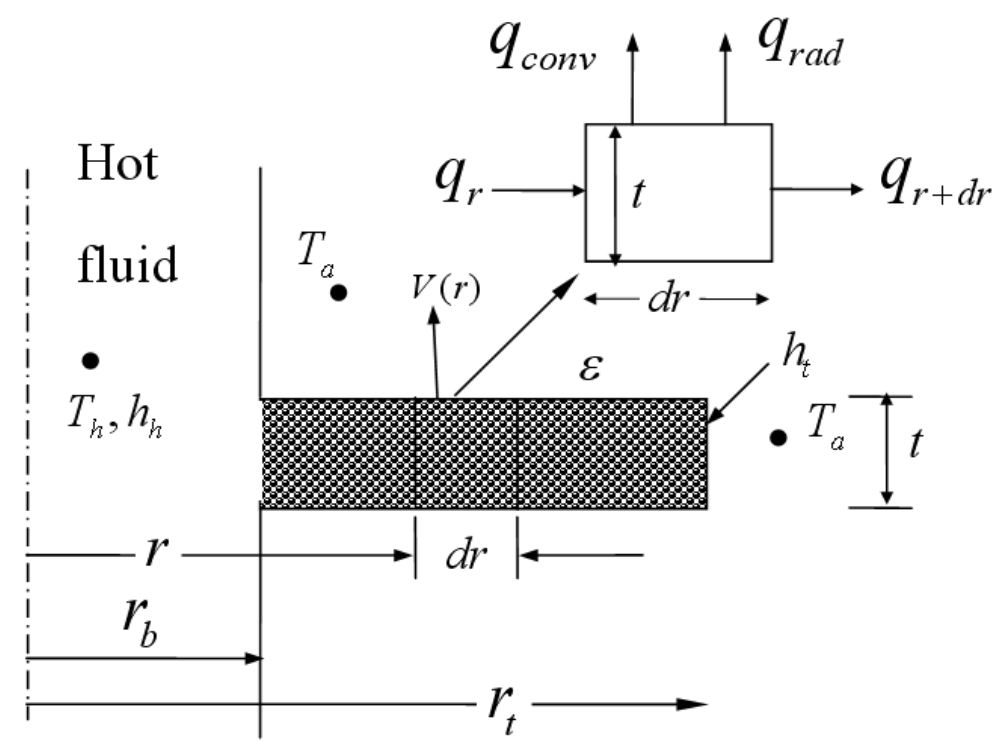

Fig.1. Porous radial fin geometry and energy balance. 
The following assumptions are made in the derivation of the energy equation:

1. the porous fin is homogeneous, isotropic and saturated with the single-phase ambient fluid,

2. the thermo physical properties of both the solid matrix and the fluid are independent of temperature,

3. the solid matrix and the fluid are in local thermodynamic equilibrium,

4. heat conduction occurs only in the radial direction and consequently there are no transverse or circumferential temperature gradients in the fin,

5. the solid-fluid interaction is described by Darcy's law,

6. the fin operates under steady state condition.

Making an energy balance on an element of the fin (Fig.1) of circumference $2 \pi r$, thickness $t$ and radial height $d r$, we get

$$
q_{r}-q_{r+d r}-q_{c o n v}-q_{\text {rad }}=0
$$

where

$$
\begin{aligned}
& q_{r}-q_{r+d r}=\frac{d}{d r}\left(k(2 \pi r t) \frac{d T}{d r}\right) d r, \\
& q_{r a d}=2 \varepsilon \sigma F_{f-a}(2 \pi r d r)\left(T^{4}-T_{a}^{4}\right), \\
& q_{c o n v}=\rho_{f} v(r)(2 \pi r d r) c_{p, f}\left(T-T_{a}\right)
\end{aligned}
$$

where Eq.(2.2) is based on the application of Fourier's law of heat conduction, Eq.(2.3) gives the radiative heat losses from the top and bottom faces of the fin and Eq.(2.4) is the rate of change of enthalpy of the buoyant fluid (infiltrate) passing through the fin. This is the rate at which the energy is removed from the fin by the buoyancy induced flow through the fin. The velocity of the buoyancy driven flow $v(r)$ at any radiation location $r$ is obtained by applying Darcy's law as follows

$$
v(r)=\frac{g \beta\left(T-T_{a}\right)}{v_{f}} .
$$

Substituting Eqs (2.2)-(2.5) into Eq.(2.1) leads to the following nonlinear ordinary differential equation governing the temperature distribution in the fin

$$
\frac{1}{r} \frac{d}{d r}\left(r \frac{d T}{d r}\right)-\frac{\rho_{f} g \beta_{f} K}{v_{f} k_{e f f} t}\left(T-T_{a}\right)^{2}-\frac{2 \varepsilon \sigma F_{f-a}}{k_{e f f} t}\left(T^{4}-T_{a}^{4}\right)=0 .
$$

We assume that

$$
k_{e f f}=k_{0}\left(1+\lambda\left(T-T_{a}\right)\right)=k_{0}\left(1+m\left(\theta-\theta_{a}\right)\right)
$$

where $k_{0}=$ effective thermal conductivity at $T_{a}$ and $m=\lambda T_{b}$.

The boundary conditions at the fin's base and at the fin's tip may be written as 


$$
\begin{aligned}
& r=r_{b}, \quad T=T_{b}, \\
& r=r_{t}, \quad \frac{d T}{d r}=0 .
\end{aligned}
$$

We introduce the following dimensionless quantities

$$
\begin{aligned}
& \theta=\frac{T}{T_{b}}, \quad \theta_{a}=\frac{T_{a}}{T_{b}}, \quad R=\frac{r}{r_{b}}, \quad K_{r}=\frac{k_{e f f}}{k_{f}}, \quad \mathrm{Da}=\frac{K}{t^{2}}, \quad \mathrm{Gr}=\frac{g \beta_{f} T_{b} t^{3}}{v_{f}^{2}}, \\
& \operatorname{Pr}=\frac{v_{f}}{\alpha_{f}}, \quad \mathrm{Ra}=\mathrm{GrPr}, \quad N c=\frac{\mathrm{DaRa}}{K_{r}}\left(\frac{r_{b}}{t}\right)^{2}=\frac{\rho_{f} g K \beta_{f} c_{p, f} r_{b}^{2} T_{b}}{v_{f} k_{e f f} t}, \\
& N r=\frac{2 \varepsilon \sigma F_{f-a} r_{b}^{2} T_{b}^{3}}{k_{\text {eff }} t},
\end{aligned}
$$

and rewrite Eqs (2.6)-(2.9) in a dimensionless form as follows

$$
\begin{aligned}
& \frac{1}{R} \frac{d}{d R}\left(R \frac{d \theta}{d R}\right)-N c \frac{\left(\theta-\theta_{a}\right)^{2}}{1+m\left(\theta-\theta_{a}\right)}-N r \frac{\left(\theta^{4}-\theta_{a}^{4}\right)}{1+m\left(\theta-\theta_{a}\right)}=0, \\
& R=1, \quad \theta(R)=1, \\
& R=R^{*}, \quad \frac{d}{d R} \theta(R)=0 .
\end{aligned}
$$

Equation (2.12) is a nonlinear ordinary differential equation. It contains two nonlinear terms. The first nonlinearity is due to the natural convective transport of energy by the infiltrate. This energy is the rate at which the enthalpy of the infiltrate increases as it flows through the porous fin. The second nonlinear term is associated with the surface radiative heat transfer from the fin to the ambient fluid which also serves as the radiation sink.

It may be noted that the parameter $N c$ is a combination of Darcy's number Da, Rayleigh number $\mathrm{Ra}$, the thermal conductivity ratio $K_{r}$ and the ratio of fin base radius to fin thickness. The parameter $\mathrm{Nr}$ indicates the role of surface radiation relative to conduction in the fin. The parameter $\theta_{a}$ is the ratio of ambient fluid temperature and the base temperature.

The heat flow through the fin, $q$, can be found by applying Fourier's law at the base of the fin giving

$$
q=-\left.k_{0}\left(1+\lambda\left(T_{b}-T_{a}\right)\right)\left(2 \pi r_{b} t\right) \frac{d T}{d r}\right|_{r=r_{b}}
$$

or a dimensionless form as 


$$
Q=\frac{q}{2 \pi k_{0} t T_{b}}=-\left(1+m\left(1-\theta_{a}\right)\right) \theta^{\prime}(1)
$$

In this paper, we solve Eq.(2.12) with boundary conditions Eqs (2.13) and (2.14) by the spectral collocation method (Darvishi, 2007).

\section{Numerical method}

To apply the spectral collocation method, we begin by transforming the domain of the problem from $\left[1, R^{*}\right]$ to $[-1,1]$ using the mapping

$$
R=\frac{R^{*}-1}{2} \xi+\frac{1+R^{*}}{2}, \quad-1 \leq \xi \leq 1
$$

Substituting Eq.(3.1) in the governing Eq.(2.12) and boundary conditions Eqs (2.13) and (2.14) gives

$$
\left(1+m\left(\theta-\theta_{a}\right)\right)\left(a_{1} \theta^{\prime}+a_{2} R \theta^{\prime \prime}\right)-a_{3}\left(\theta-\theta_{a}\right)^{2}-a_{4}\left(\theta^{4}-\theta_{a}^{4}\right)=0,
$$

subject to

$$
\theta(-1)=1, \quad \theta^{\prime}(1)=0
$$

where the primes denote differentiation with respect to $\xi$ and

$$
a_{1}=\frac{2}{R^{*}-1}, \quad a_{2}=\frac{4}{\left(R^{*}-1\right)^{2}}, \quad a_{3}=N c R, \quad a_{4}=N r R
$$

we use the spectral collocation method to solve Eqs (3.2) and (3.3). The unknown function $\theta(\xi)$ is approximated as a truncated series of Chebyshev polynomials of the form

$$
\theta(\xi) \approx \sum_{k=0}^{N} \hat{\theta}_{k} T_{k}\left(\xi_{j}\right), \quad j=0,1, \ldots, N
$$

where $T_{k}$ is the $k$-th Chebyshev polynomial, $\hat{\theta}_{k}$, are coefficients and $\xi_{0}, \xi_{1}, \ldots, \xi_{N}$ are Gauss-Lobatto collocation points (see Darvishi, 2007) defined by

$$
\xi_{j}=\cos \frac{\pi j}{N}, \quad j=0,1, \ldots, N
$$

Derivatives of the functions $\theta(\xi)$ at the collocation points are represented as

$$
\frac{d^{s} \theta}{d \xi^{s}}=\sum_{k=0}^{N} \mathrm{D}_{k j}^{s} \theta\left(\xi_{j}\right)
$$


where $s$ is the order of differentiation and $\mathrm{D}$ is the Chebyshev spectral differentiation matrix and the entries of the matrix for $j=0,1, \ldots, N, k=0,1, \ldots, N / 2$, are

$$
\begin{cases}\mathrm{D}_{k j}=-\frac{c_{k}}{2 c_{j}} \frac{(-1)^{j+k}}{\mathrm{D}_{k k}=-\frac{1}{2} \cos \left(\frac{k \pi}{N}\right)\left(1+\cot ^{2}\left(\frac{k \pi}{N}\right)\right),}, & j \neq k, \\ \mathrm{D}_{00}=-d_{N N}=\frac{2 N^{2}+1}{6}, & k \neq 0, \\ \mathrm{D}_{k j}=-d_{N-k, N-j}, & k=\frac{N}{2}+1, \ldots, N .\end{cases}
$$

Substituting Eqs (3.4)-(3.7) in Eqs (3.2) and (3.3) gives the following equations

$$
\left(1+m\left(\Theta-\theta_{a}\right)\right)\left(a_{1} \mathrm{D} \Theta+a_{2} R \mathrm{D}^{2} \Theta\right)-a_{3}\left(\Theta-\theta_{a}\right)^{2}-a_{4}\left(\Theta^{4}-\theta_{a}^{4}\right)=0,
$$

subject to

$$
\hat{\theta}_{0}=1, \quad \sum_{k=0}^{N} \mathrm{D}_{N k} \hat{\theta}_{N}=0
$$

where

$$
\Theta=\left\{\hat{\theta}_{0}, \hat{\theta}_{1}, \ldots, \hat{\theta}_{N}\right\}^{T}, \quad a_{r}=\operatorname{diag}\left(\left[a_{r}\left(\xi_{0}\right), a_{r}\left(\xi_{1}\right), \ldots, a_{r}\left(\xi_{N-1}\right), a_{r}\left(\xi_{N}\right)\right]\right), \quad r=3,4 .
$$

Equations (3.8) and (3.9) form a system of nonlinear algebraic equations and are solved using the classical Newton iteration method.

\section{Results and discussion}

Figure 2 shows the effect of natural convective heat loss on the temperature distribution in the fin when the radiation heat loss, the environment temperature, and the ratio of outer to inner radius are kept fixed. As the buoyancy effects become stronger, i.e., $N c$ increases, the local temperature in the fin decreases. This behavior is synonymous with the well known behavior of the solid radial fin. The decrease in the local temperature is accompanied by the increase in the base heat flow as will be seen later. When the radiation parameter $\mathrm{Nr}$ is allowed to vary as in Fig.3, the local fin temperature decreases because of the increasing strength of radiative heat exchange between the exposed surface of the fin and the ambient.

Figure 4 shows that as the ambient temperature decreases, it causes the local fin temperature to decrease. These results pertain to the circumstance when the buoyancy induced natural convection is much stronger $(N c=10)$ than the surface radiation transport $(N r=1)$. The quantity of more practical importance is the heat flow through the base of the fin, $-\theta^{\prime}(1)$, given by Eq.(2.16). The results appear in Figs 6-8 and cover a wide range of values of the parameters $N c, N r$ and $\theta_{a}$ for fin geometries with $R^{*}=1.75$ and 2.5. These values of $R^{*}$ are often used to design the cooling fins attached to the cylindrical surfaces of combustion chambers and for improving the heat dissipation from the gas-side of the heat exchanger tubes. The results in Figs 6-8 
confirm the expectation that as the natural convection and radiation effects increase in strength either individually or in concert, the base heat flow increases. We believe that the results presented in Figs 6-8 can be used to evaluate the thermal performance of porous radial fins under a variety of operational conditions.

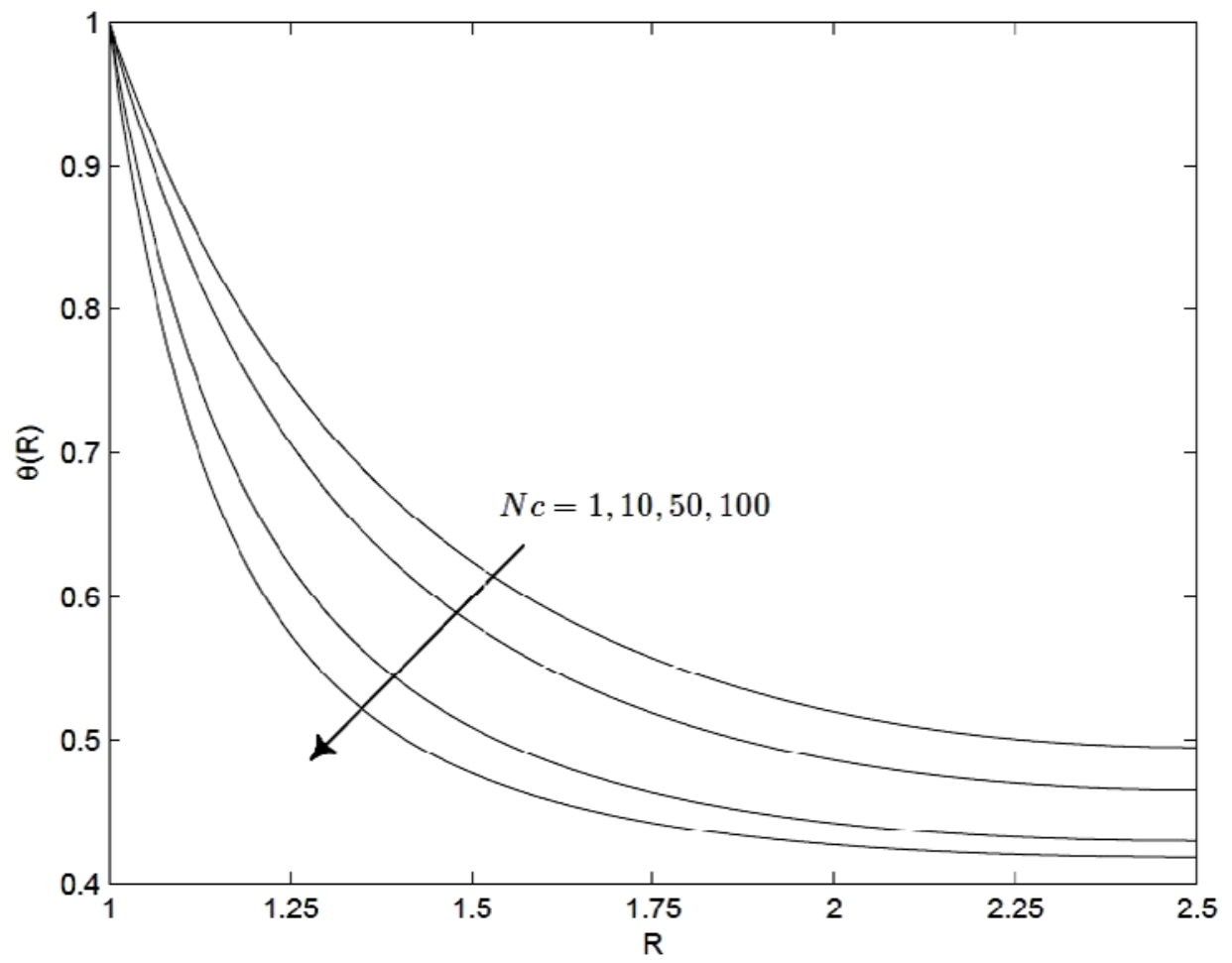

Fig.2. Temperature distributions at $\theta_{a}=0.4, N r=5, m=0.5$ and $R^{*}=2.5$, for different values of $N c$.

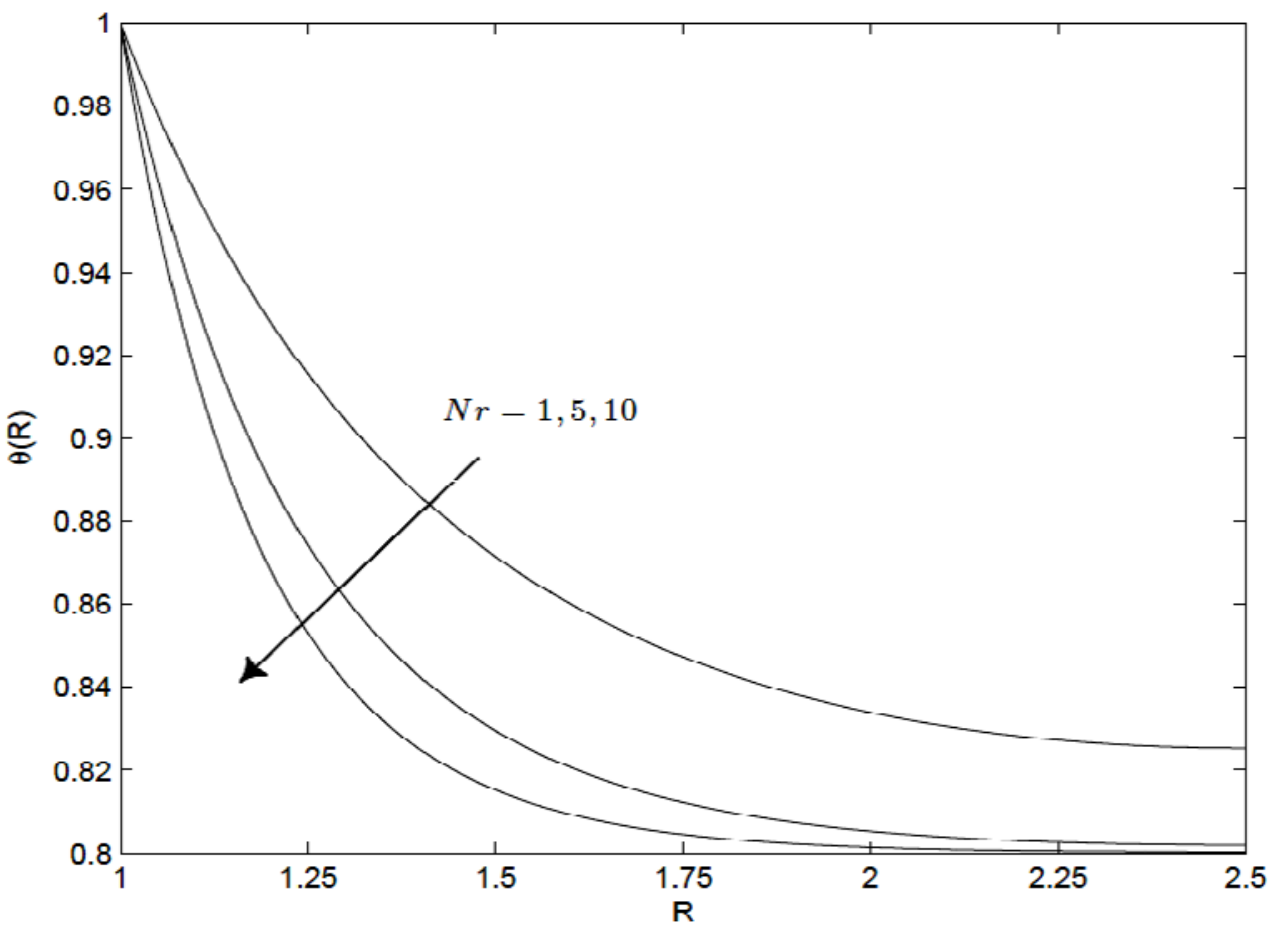

Fig.3. Temperature distributions at $\theta_{a}=0.8, N c=10, m=0.1$ and $R^{*}=2.5$, for different values of $N r$. 


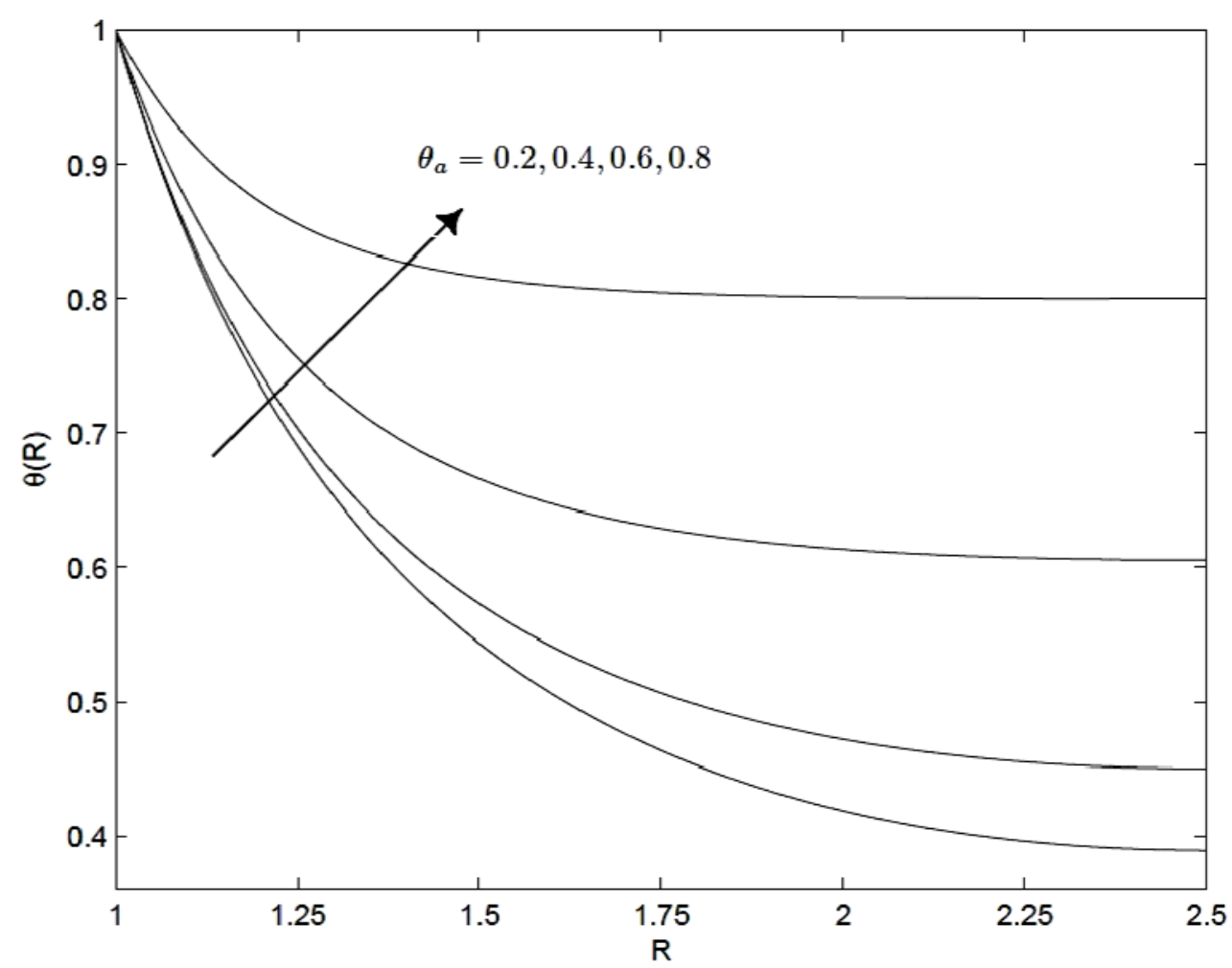

Fig.4. Temperature distributions at $N r=10, N c=1, m=1$ and $R^{*}=2.5$, for different values of $\theta_{a}$.

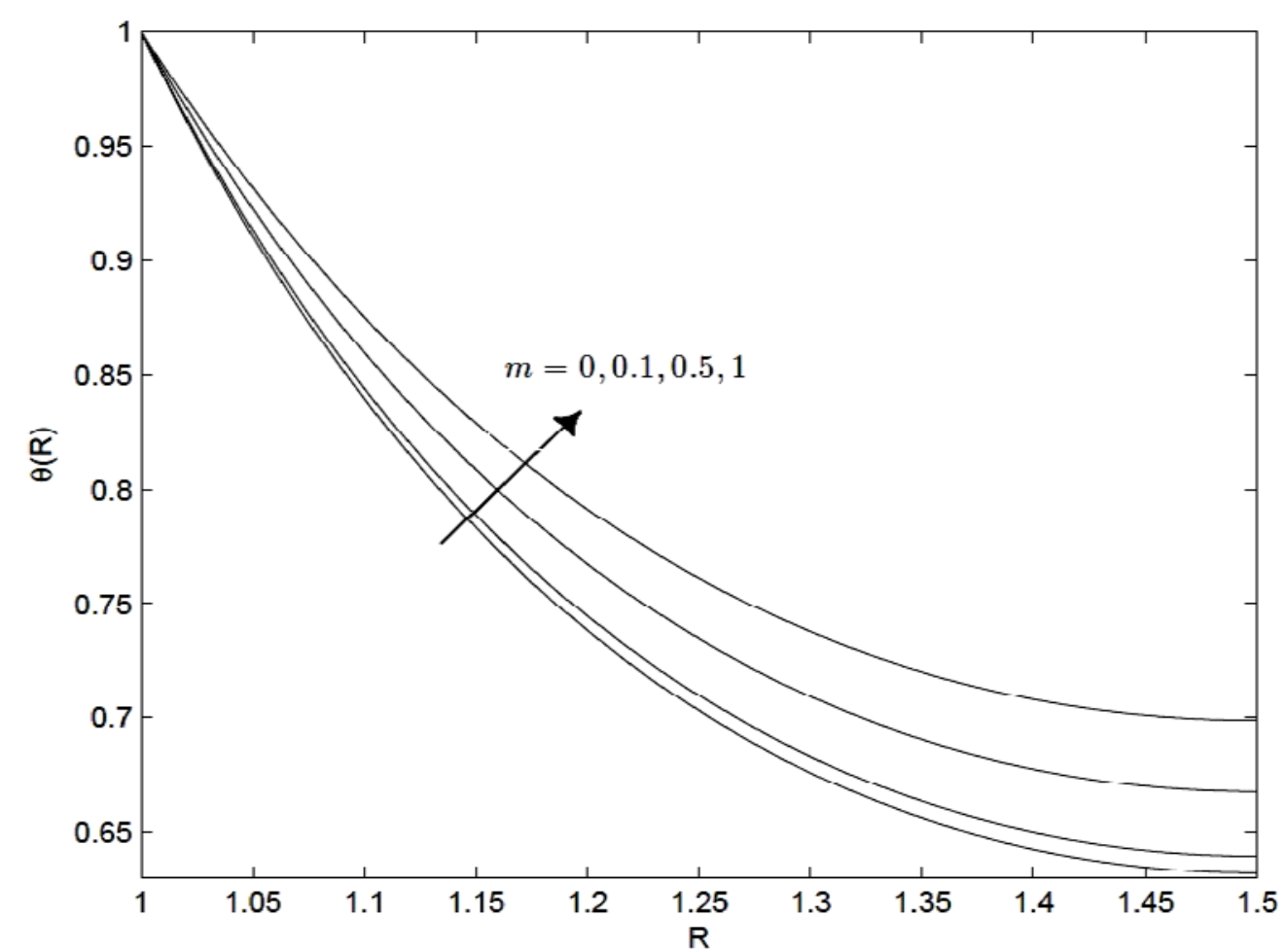

Fig.5. Temperature distributions at $N r=1, N c=10, \theta_{a}=0.2$ and $R^{*}=1.5$, for different values of $m$. 


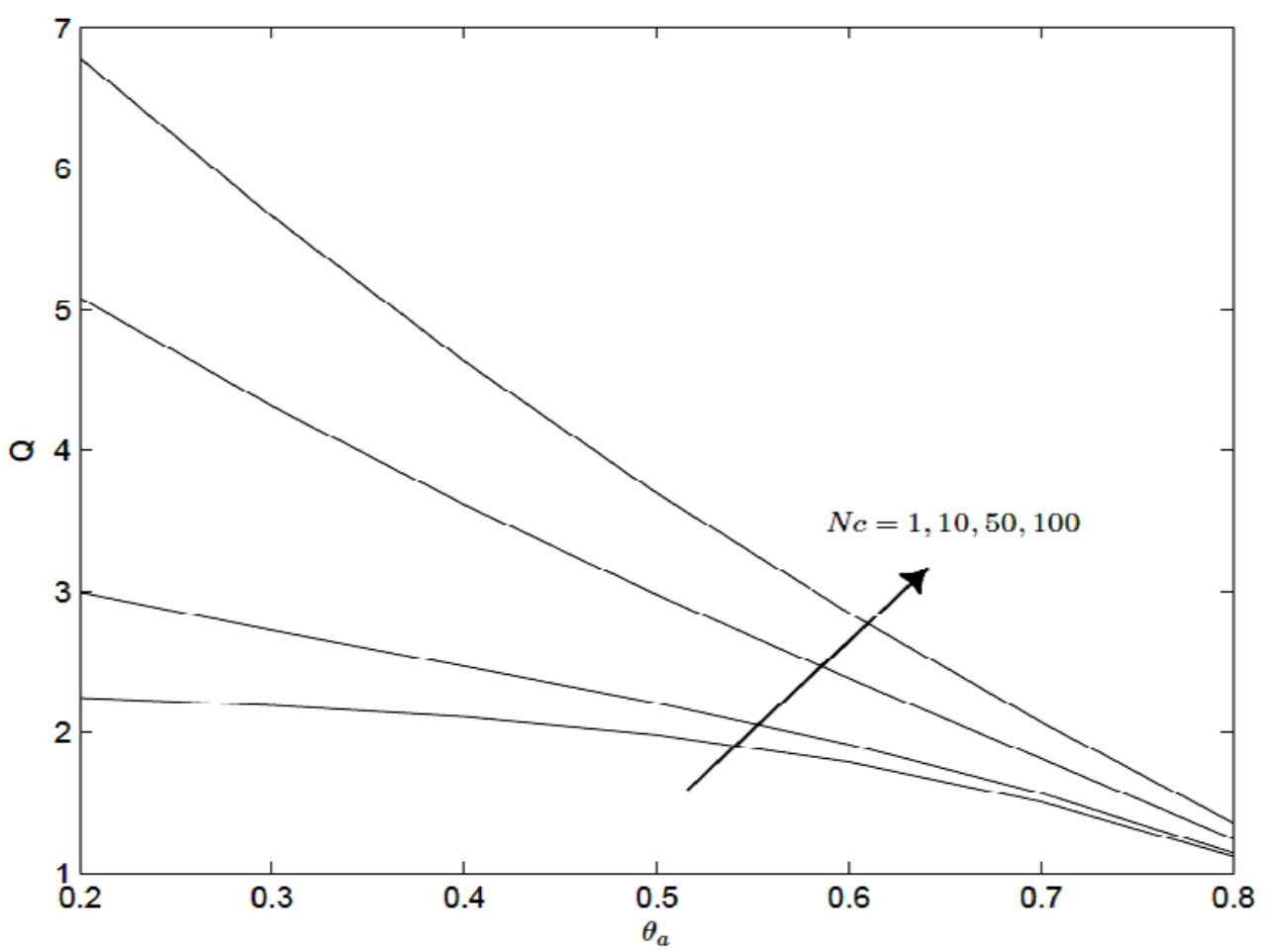

Fig.6. Dimensionless base heat flow effect of natural convection and ambient temperature when $N r=10, m=0.1, R^{*}=1.5$.

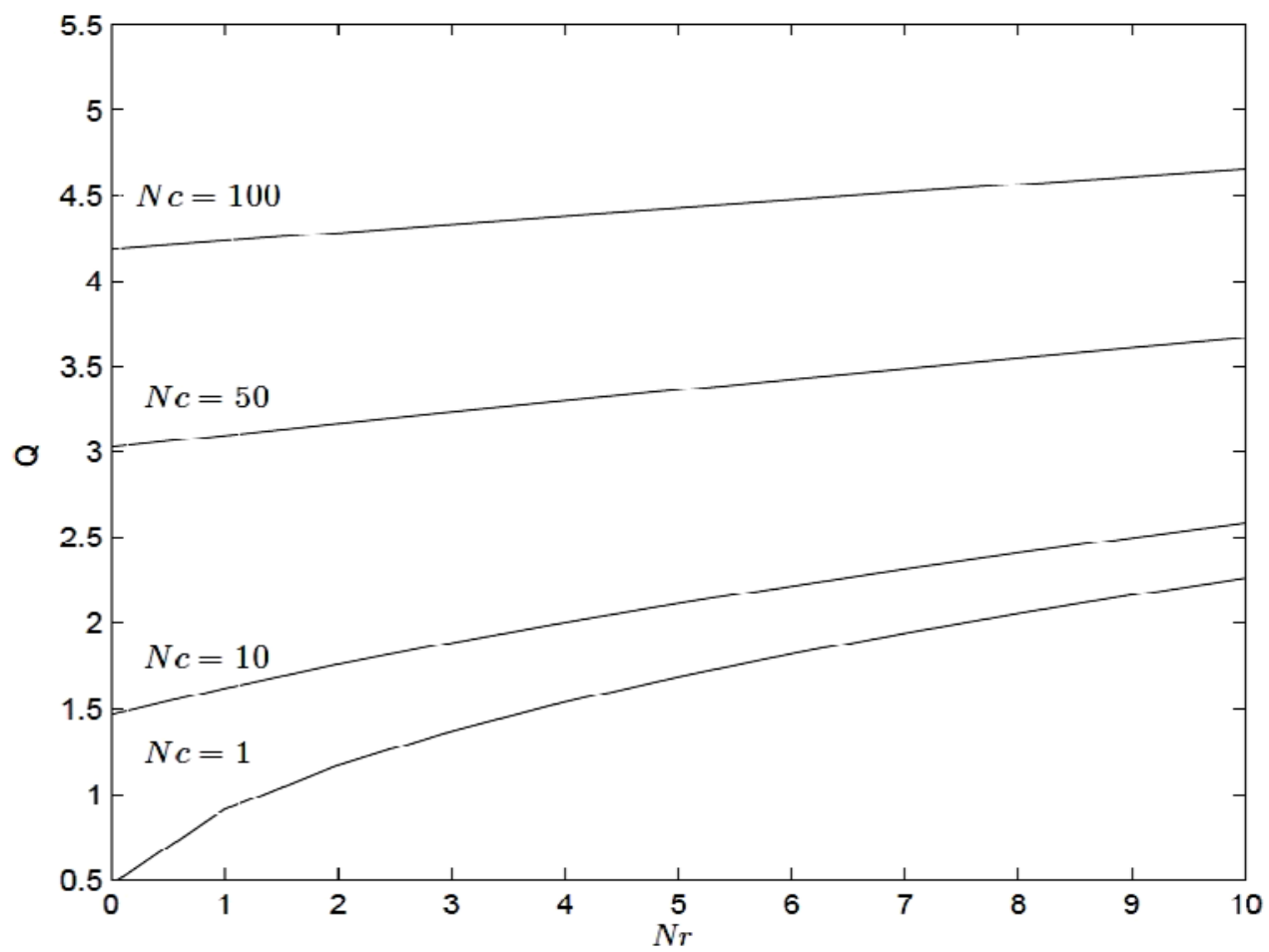

Fig.7. Dimensionless base heat flow effect of natural convection and radiation when $\theta_{a}=0.4, m=0.1, R^{*}=2.5$. 


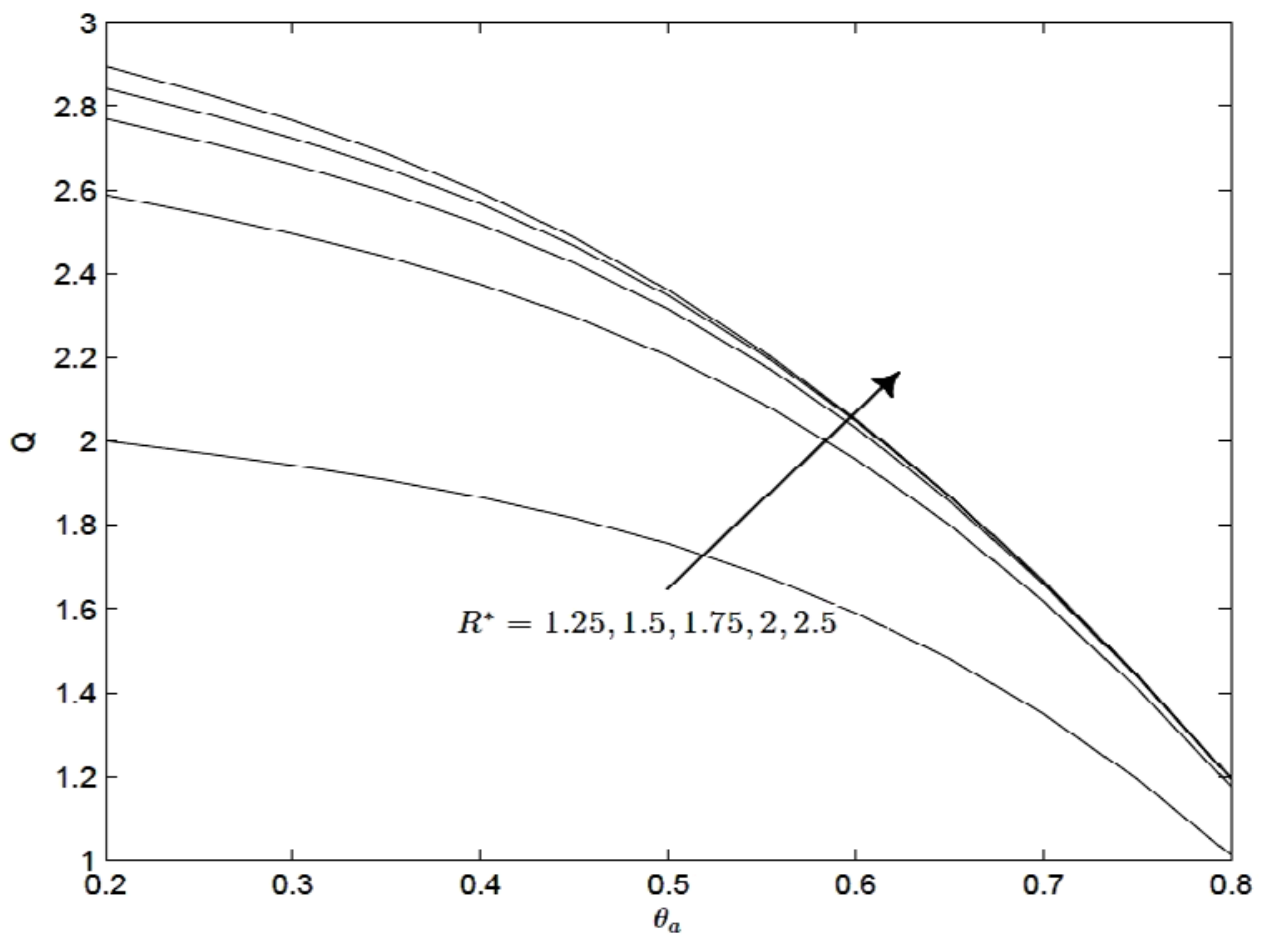

Fig.8. Dimensionless base heat flow effect of fin radial parameter and ambient temperature when $N c=1, N r=10, m=0.5$.

\section{Concluding remarks}

The thermal analysis of a radial porous fin for convection and radiation heat transfer has been performed here. A second order non-linear ordinary differential equation has been derived as the governing equation for this problem. It has been solved using the spectral method. It is also found that all geometric and flow parameters influencing the temperature distribution have been grouped into three parameters, namely, $N c, N r$ and $m$. The effect of these parameters on heat transfer has been investigated. It was found that increasing $\mathrm{Nr}$ by increasing either Da or Ra increases heat transfer from the fin. In addition, increasing the radiation parameter $\mathrm{Nr}$ also increases heat transfer from the radial fin.

\section{Nomenclature}

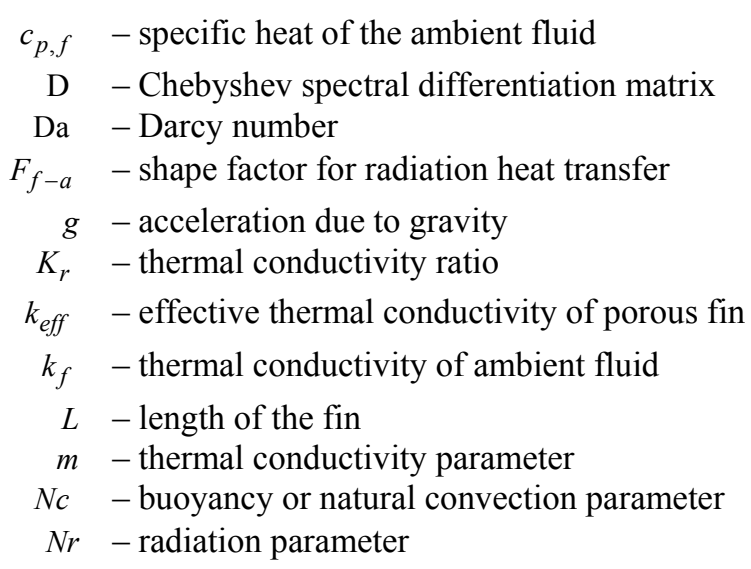




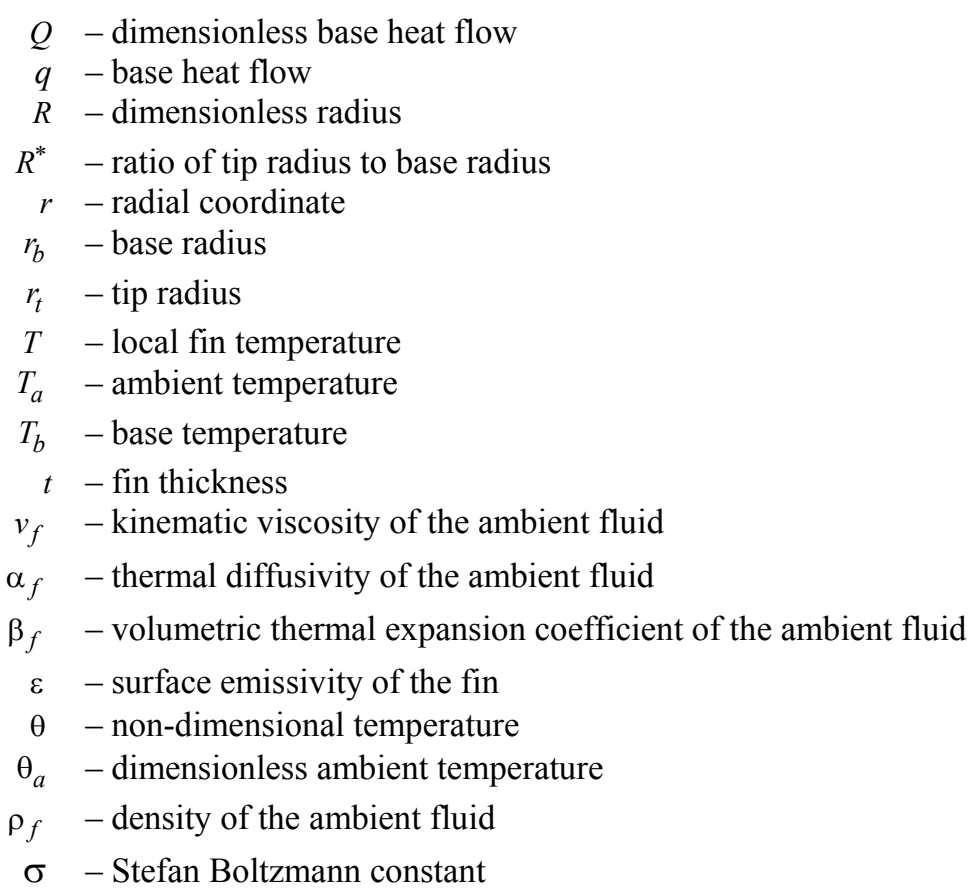

\section{References}

Abu-Hijleh (2003): Enhanced forced convection heat transfer from a cylinder using permeable fins. - ASME J. Heat Transfer, vol.125, pp.804-811.

Darvishi M.T. (2007): Spectral collocation method and Darvishis preconditionings for Tchebychev-Gauss-Lobatto points. - International Mathematical Forum, vol.2(6), pp.263-272.

Gorla R.S.R. and Bakier A.Y. (2011): Thermal analysis of natural convection and radiation in porous fins. - Int. Commun. Heat Mass Transfer, vol.38, pp.638-645.

Kiwan S. (2006): Thermal analysis of natural convection in porous fins. - Transport in Porous Media, vol.67, pp.17-29.

Kundu B. and Bhanja D. (2010): Performance and optimization analysis of a constructal T-shaped fin subject to variable thermal conductivity and convective heat transfer coefficient. - Int. J. Heat Mass Transfer, vol.53 pp.254267.

Lorenzini G. and Moretti S. (2007): Numerical analysis on heat removal from Y-shaped fins: efficiency and volume occupied for a new approach to performance optimization. - Int. J. Thermal Science, vol.46, pp.573-579.

Mobedi M. and Sunden B. (2006): Natural convection heat transfer from a thermal heat source located in a vertical plate fin. - Int. J. Heat Mass Transfer, vol.33, pp.943-950.

Nguyen A. and Aziz A. (1992): The heat transfer rate from convecting-radiating fins for different profile shapes. - Heat and Mass Transfer, vol.27 pp.67-72.

Yu L.T. and Chen C.K. (1999): Optimization of circular fins with variable thermal parameters. - J. Franklin Institute, vol.336 pp.77-95.

Received: April 9, 2013

Revised: August 26, 2013 